\title{
Influence of left ventricular relaxation on the pressure half time of aortic regurgitation
}

\author{
S F de Marchi, S Windecker, B C Aeschbacher, C Seiler
}

\begin{abstract}
Background-The severity of aortic regurgitation can be estimated using pressure half time (PHT) of the aortic regurgitation flow velocity, but the correlation between regurgitant fraction and PHT is weak.
\end{abstract}

Aim-To test the hypothesis that the association between PHT and regurgitant fraction is substantially influenced by left ventricular relaxation.

Methods-In 63 patients with aortic regurgitation, subdivided into a group without $(n=22)$ and a group with $(n=41)$ left ventricular hypertrophy, regurgitant fraction was calculated using the difference between right and left ventricular cardiac outputs. Left ventricular relaxation was assessed using the early to late diastolic Doppler tissue velocity ratio of the mitral annulus (E/ADTI), the E/A ratio of mitral inflow (E/AM), and the $E$ deceleration time (E-DT). Left ventricular hypertrophy was assessed using the $M$ mode derived left ventricular mass index.

Results-The overall correlation between regurgitant fraction and PHT was weak $(r=0.36, p<0.005)$. In patients without left ventricular hypertrophy, there was a significant correlation between regurgitant fraction and PHT $(r=0.62$, p $<0.005)$, but not in patients with left ventricular hypertrophy. In patients with a left ventricular relaxation abnormality (defined as E/ADTI $<1$, E/AM $<$ age corrected lower limit, E-DT $\geqslant 220 \mathrm{~ms}$ ), no associations between regurgitant fraction and PHT were found, whereas in patients without left ventricular relaxation abnormalities, the regurgitant fraction to PHT relations were significant (normal E/AM: $r=0.57, \quad$ p $=0.02 ; \quad$ E-DT $<220 \quad$ ms: $r=0.50, \mathrm{p}<0.001 ;$ E/ADTI $<1: r=0.57$, $\mathrm{p}=\mathbf{0 . 0 2 )}$.

Conclusions-Only normal left ventricular relaxation allows a significant decay of PHT with increasing aortic regurgitation severity. In abnormal relaxation, which is usually present in left ventricular hypertrophy, wide variation in prolonged backward left ventricular filling may cause dissociation between the regurgitant fraction and PHT. Thus the PHT method should only be used in the absence of left ventricular relaxation abnormalities. (Heart 1999;82:607-613)

Keywords: aortic regurgitation; left ventricular relaxation; pressure half time
Although severe aortic regurgitation should be corrected when more than mild symptoms develop, there is compelling evidence that patients with this condition should undergo valve replacement before the onset of permanent left ventricular damage, even in the absence of symptoms. ${ }^{1-3}$ The " 55 rule" has been useful in gauging the timing of surgery in severe aortic regurgitation - that is, it ought to be performed before the ejection fraction falls below $55 \%$ or the end systolic left ventricular dimension exceeds $55 \mathrm{~mm}^{1-3}$ The rule requires a correct classification of the severity of aortic regurgitation, a task which is now mainly performed using Doppler echocardiography. A standard method for the measurement of aortic regurgitation is the determination of the pulmonary to systemic cardiac output ratio. ${ }^{45}$ However, this is very time consuming and often not applicable because good ultrasound echo quality is needed from different acoustic windows. The aortic regurgitation Doppler pressure half time (PHT), a widely used parameter for assessing aortic regurgitation by continuous wave Doppler ultrasound, is more easily obtained. ${ }^{67}$ The PHT method is based on the observation that the velocity decay of the aortic regurgitation Doppler spectrum is linearly related to regurgitation severity. ${ }^{7-10}$ However, the method has failed to differentiate between mild, moderate, and severe aortic regurgitation because of a considerable PHT overlap between these severity groups. ${ }^{10}$ This suggests that variables other than the aortic regurgitation orifice area, for example ventricular and vascular factors, exert a substantial influence on PHT (fig 1).

The influence of left ventricular compliance on the PHT has been modelled in vitro. ${ }^{811}$ Restrictive ventricular physiology has been repeatedly shown to account for shortened PHT in humans. ${ }^{79}$ However, the influence of left ventricular relaxation on aortic regurgitation velocity decay has never been investigated directly in humans. The purpose of this study was therefore to determine the influence of left ventricular relaxation on the relation between quantitatively assessed aortic regurgitation severity and the pressure half time of the regurgitant jet in patients with mild to severe aortic regurgitation.

\section{Methods}

STUDY PATIENTS

We prospectively studied 63 patients (mean (SD) age, 55 (17) years) with aortic regurgitation referred for echocardiographic examination between January 1997 and February 1998. Patients with more than slight regurgita- 

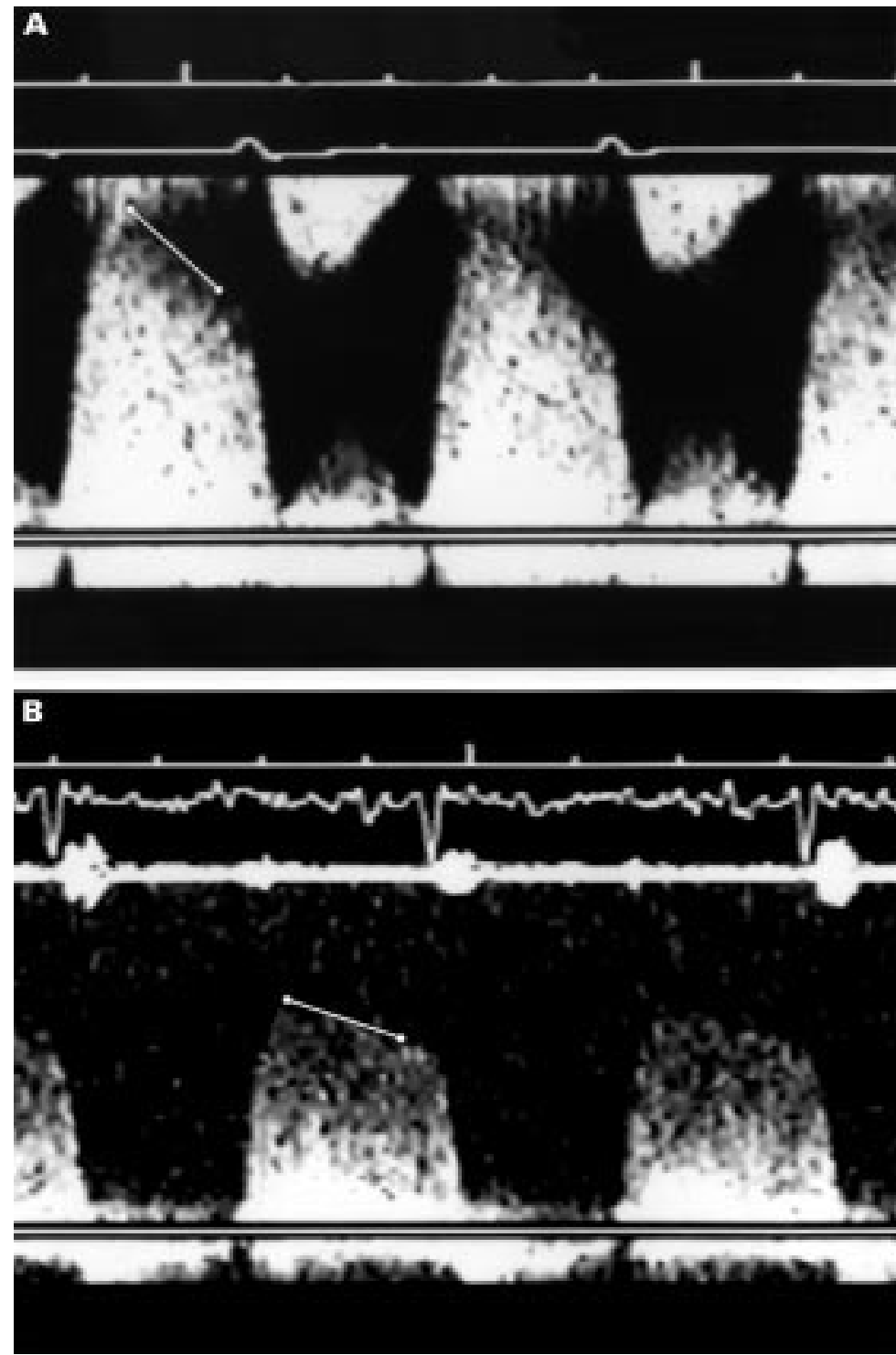

Figure 1 (A) Representative example of aortic regurgitation Doppler velocity spectrum in a 35 year old patient with regurgitant fraction of $56 \%$, a pressure half time of aortic regurgitation of $260 \mathrm{~ms}$, and a left ventricular mass index of $106 \mathrm{~g} / \mathrm{m}^{2}$. (B) Aortic regurgitation Doppler velocity spectrum in a 54 year old patient with regurgitant fraction of $53 \%$, a pressure half time of $528 \mathrm{~ms}$, and a left ventricular mass index of $185 \mathrm{~g} / \mathrm{m}^{2}$.

tion or stenosis of any valve other than the aortic valve, and patients with intracardiac or extracardiac shunts were excluded. Patients were divided into two groups: those with and those without left ventricular hypertrophy.

ECHOCARDIOGRAPHIC METHODS

All echocardiographic studies were performed on an Acuson Sequoia or Acuson XP128 ultrasonography system (Acuson Inc, Mountain View, California, USA) equipped with Doppler tissue imaging (DTI). DTI is a modification of the conventional Doppler technology, in which tissue derived, slow motion Doppler velocity signals $(<10 \mathrm{~cm} / \mathrm{s})$ rather than blood flow Doppler signals $(10-100 \mathrm{~cm} / \mathrm{s})$ can be displayed either as time-velocity tracings or as cross sectional or $\mathrm{M}$ mode colour images. ${ }^{12-15}$ All patients were examined in a left lateral supine position. The examinations were recorded on VHS videotapes for off-line analysis.
Measurement of aortic regurgitation

The aortic regurgitant fraction was calculated using the measurement of the pulmonary to systemic cardiac output ratio (Qp/Qs method).

The systemic and pulmonary cardiac output

$$
R F(\%)=\left(-\frac{Q p}{Q s}\right) \times 100
$$

(Qs and Qp, respectively) were determined from the diameters of the right and left ventricular outflow tracts, the Doppler derived flow velocity time integrals across the corresponding outflow tracts, and the heart rate. The regurgitant fraction was assessed by two independent investigators. For the measurement of the pressure half time of the aortic regurgitation Doppler spectrum, visual linear fitting of the continuous wave spectral velocity decay was performed. The PHT of aortic regurgitation was calculated as the time required for the peak velocity to decay to $1 / 2$. The length of the colour Doppler regurgitant jet in the parasternal long axis and apical four chamber view was used for semiquantitative assessment of the regurgitation severity as follows: mild, jet limited to the left ventricular outflow tract; moderate, jet exceeding the left ventricular outflow tract but not exceeding the papillary muscle level; severe, jet exceeding the papillary muscle level.

Assessment of left ventricular relaxation

The transmitral inflow pattern (that is, the "conventional" method for assessing left ventricular relaxation) was obtained from pulsed wave Doppler recordings with the sample volume placed at the tips of the mitral valve leaflets. Measurements included the early to late peak velocity ratio of transmitral flow (E/AM), the deceleration time of early transmitral filling (E-DT, ms), and the isovolumic relaxation time (IVRT, ms). An age adjusted cut off point was used to separate normal from abnormally low E/AM, ${ }^{16}$ whereas for the E-DT, a cut off point of $220 \mathrm{~ms}$ was used. The right upper pulmonary vein flow signal was recorded using pulsed wave Doppler. Measurements included the systolic to diastolic peak flow velocity ratio $(\mathrm{S} / \mathrm{D})$, the atrial reverse peak flow velocity (peak Arev, $\mathrm{cm} / \mathrm{s}$ ), and the duration of atrial reverse flow (Adur, ms).

In addition, Doppler tissue imaging (DTI) of the mitral annulus was performed to assess left ventricular relaxation. ${ }^{12}$ From the apical four chamber view, a pulsed wave Doppler sample volume was placed at the septal portion of the mitral annulus. The ratio of the early to late peak diastolic annulus velocity (E/ADTI) was calculated. This method has been reported to be a relatively load independent method for assessing left ventricular relaxation, reflecting predominantly the muscular component of relaxation. ${ }^{17}$ An E/ADTI ratio of less than 1 was considered to indicate impaired left ventricular relaxation. Images of aortic regurgitation were digitised on a SunSparc Station computer, and the left ventricular relaxation constant Tau was assessed as previously described. ${ }^{18}$ 
Table 1 Patients characteristics

\begin{tabular}{lcccc}
\hline & $\begin{array}{c}\text { Total } \\
(n=63)\end{array}$ & $\begin{array}{c}\text { LVH } \\
(n=41)\end{array}$ & $\begin{array}{c}\text { No LVH } \\
(n=22)\end{array}$ & p Value \\
\hline Age (years) & $55(16)$ & $58(15)$ & $49(18)$ & 0.04 \\
Male/female & $40 / 23$ & $24 / 17$ & $16 / 6$ & NS \\
Heart rate (beats/min) & $68(13)$ & $69(13)$ & $70(13)$ & NS \\
Systolic blood pressure (mm Hg) & $136(26)$ & $139(30)$ & $131(15)$ & NS \\
Diastolic blood pressure (mm Hg) & $76(16)$ & $74(17)$ & $76(16)$ & NS \\
Causes of aortic regurgitation & $13(21 \%)$ & $9(22 \%)$ & $4(18 \%)$ & NS \\
$\quad$ Degenerative & $20(32 \%)$ & $12(29 \%)$ & $8(36 \%)$ & NS \\
Idiopathic & $9(14 \%)$ & $6(15 \%)$ & $3(14 \%)$ & NS \\
Aortic root dilatation & $8(13 \%)$ & $6(15 \%)$ & $2(9 \%)$ & NS \\
$\quad$ Bicuspid valve & $4(6 \%)$ & $3(7 \%)$ & $1(5 \%)$ & NS \\
$\quad$ Infective endocarditis & $9(14 \%)$ & $5(12 \%)$ & $4(18 \%)$ & NS \\
Other causes & $12(19 \%)$ & $8(20 \%)$ & $4(18 \%)$ & NS \\
Concomitant diseases & $12(19 \%)$ & $9(22 \%)$ & $3(14 \%)$ & NS \\
$\quad$ Coronary artery disease & & & & \\
$\quad$ Systemic hypertension & $8(13 \%)$ & $5(12 \%)$ & $3(14 \%)$ & NS \\
Drugs & $12(19 \%)$ & $10(24 \%)$ & $2(9 \%)$ & NS \\
$\quad$ Calcium antagonists & $11(17 \%)$ & $8(20 \%)$ & $3(14 \%)$ & NS \\
$\quad$ Blockers & $2(3 \%)$ & $2(5 \%)$ & $0(0 \%)$ & NS \\
$\quad$ ACE inhibitors & Nitrates & & & \\
\hline
\end{tabular}

Data are presented as mean (SD) or number (\%) of patient group.

ACE, angiotensin converting enzyme; LVH, left ventricular hypertrophy.

Table 2 Echocardiographic data

\begin{tabular}{|c|c|c|c|}
\hline & $\begin{array}{l}L V H \\
(n=41)\end{array}$ & $\begin{array}{l}\text { No LVH } \\
(n=22)\end{array}$ & $p$ Value \\
\hline \multicolumn{4}{|l|}{ Colour Doppler assessment of aortic regurgitation } \\
\hline Mild aortic regurgitation & $29(71 \%)$ & $18(82 \%)$ & NS \\
\hline Moderate aortic regurgitation & $5(12 \%)$ & $3(14 \%)$ & NS \\
\hline Severe aortic regurgitation & $7(17 \%)$ & $1(5 \%)$ & NS \\
\hline Left ventricular end diastolic diameter (mm) & $53(9)$ & $50(6)$ & NS \\
\hline Left ventricular end systolic diameter ( $\mathrm{mm}$ ) & $35(11)$ & $33(6)$ & NS \\
\hline End diastolic diameter of ascending aorta $(\mathrm{mm})$ & $34(5)$ & $36(6)$ & NS \\
\hline Left ventricular ejection fraction (\%) & $63(12)$ & $68(7)$ & NS \\
\hline Left ventricular mass index $\left(\mathrm{g} / \mathrm{m}^{2}\right)$ & $171(53)$ & $107(15)$ & $<0.0001$ \\
\hline Mean aortic gradient $(\mathrm{mm} \mathrm{Hg})$ & $11(11)$ & $10(10)$ & NS \\
\hline Aortic stenosis & $7(17 \%)$ & $5(23 \%)$ & NS \\
\hline \multicolumn{4}{|l|}{ Assessment of left ventricular diastolic function } \\
\hline $\mathrm{E} / \mathrm{AM}$ ratio & $0.9(0.5)$ & $1.4(1.2)$ & 0.02 \\
\hline Isovolumic relaxation time $(\mathrm{ms})$ & $93(26)$ & $83(30)$ & NS \\
\hline E deceleration time (ms) & $241(85)$ & $197(47)$ & 0.03 \\
\hline Pulmonary vein $S / D$ ratio & $1.7(1.3)$ & $1.1(0.4)$ & 0.06 \\
\hline Pulmonary vein peak A wave $(\mathrm{cm} / \mathrm{s})$ & $30(8)$ & $33(15)$ & NS \\
\hline Pulmonary vein A wave duration (ms) & $180(47)$ & $156(40)$ & 0.08 \\
\hline $\mathrm{E} / \mathrm{ADTI}$ ratio & $0.78(0.40)$ & $1.04(0.45)$ & 0.04 \\
\hline Calculated left ventricular relaxation constant $\tau$ & $55(19)$ & $57(22)$ & NS \\
\hline
\end{tabular}

Data are presented as mean (SD) or number (\%) of patient group.

$\mathrm{E} / \mathrm{ADTI}$, early to late mitral annulus peak motion velocity ratio; $\mathrm{E} / \mathrm{AM}$, early to late transmitral peak velocity flow ratio; $S / D$, systolic to diastolic peak flow velocity ratio.

\section{Left ventricular mass assessment}

Left ventricular mass index was calculated in each patient using $M$ mode echocardiography. The calculation was performed according to the conventions of the American Society of Echocardiography (ASE).${ }^{19}{ }^{20}$ The cut off point dividing left ventricular hypertrophy from normal left ventricular mass index was $109 \mathrm{~g} / \mathrm{m}^{2}$ for women and $134 \mathrm{~g} / \mathrm{m}^{2}$ for men. ${ }^{19}{ }^{21}$ The end diastolic diameter of the ascending aorta was measured in the parasternal long axis from an $\mathrm{M}$ mode echocardiogram.

\section{STATISTICAL ANALYSIS}

Demographic, clinical, and echocardiographic values are expressed as mean (SD). For comparisons of continuous values between the study groups, an unpaired two tailed Student's $t$ test was performed. A $\chi^{2}$ test was used for comparison of categorical variables between the study groups. Linear regression analysis was performed to assess the relation between aortic regurgitation pressure half time and regurgitant fraction. A p value of less than 0.05 was considered statistically significant. The interobserver variability of the regurgitant frac- tion was calculated as the standard error of estimate (SEE) of the correlation between the two observers' values.

\section{Results}

PATIENTS

The characteristics of the patients studied are given in table 1 . No significant differences were observed between the groups in relation to sex, heart rate, blood pressure, or cause of aortic regurgitation.

ECHOCARDIOGRAPHIC DATA

Echocardiographic data are given in table 2. Internal left ventricular dimensions and left ventricular ejection fraction were not statistically different between the groups. Patients with left ventricular hypertrophy were older than those without $(p=0.04)$. The $E / A$ ratios of transmitral filling and mitral annular motion velocities, and the pulmonary vein systolic to diastolic peak velocity ratio (S/D), were lower in the group without left ventricular hypertrophy $(p<0.05)$, while the pulmonary vein A wave and the $\mathrm{E}$ deceleration time (E-DT) were slower and shorter, respectively, in the group without hypertrophy $(\mathrm{p}<0.05)$. No statistically significant differences were found for the other diastolic variables, such as isovolumic relaxation time (IVRT) and left ventricular relaxation constant $(\tau)$, although there was a trend indicating impaired left ventricular relaxation in the group with left ventricular hypertrophy. The pulmonary vein A wave duration tended to be high even in the group without left ventricular hypertrophy, compared with the normal values published by Gentile et al..$^{22}$

INTEROBSERVER VARIABILITY OF THE REGURGITANT FRACTION

The regurgitant fraction of the aortic regurgitation, according to the calculation outlined above, was assessed from the video sequences by two experienced independent investigators. The interobserver variability (SEE) of the regurgitant fraction was $9 \%$ and the mean difference between the two observers was $2 \%$; the correlation equation was: $\mathrm{y}=0.07+0.87 \mathrm{x}$ $(r=0.75, \mathrm{p}<0.0001)$.

AORTIC REGURGITANT FRACTION TO PRESSURE HALF TIME RELATION

There was a significant but weak overall correlation between the regurgitant fraction and the PHT $(\mathrm{PHT}=611-3.15 \times$ regurgitant fraction; $r=0.36, \mathrm{p}<0.005)$. In patients without left ventricular hypertrophy, a good correlation was found $(\mathrm{PHT}=662-5.96 \times$ regurgitant fraction; $r=0.62, \mathrm{p}<0.005$, fig 2 ), whereas in the presence of left ventricular hypertrophy, no correlation could be observed.

In patients with signs of impaired left ventricular relaxation, such as an abnormally low transmitral $\mathrm{E} / \mathrm{A}$ ratio, $\mathrm{E}$ deceleration time above $220 \mathrm{~ms}$, or an E/A ratio of the mitral annular motion below 1, no statistically significant correlation between the regurgitant fraction and the PHT was observed. In the absence of these abnormalities, however, significant correlations were found (for normal E/AM: 


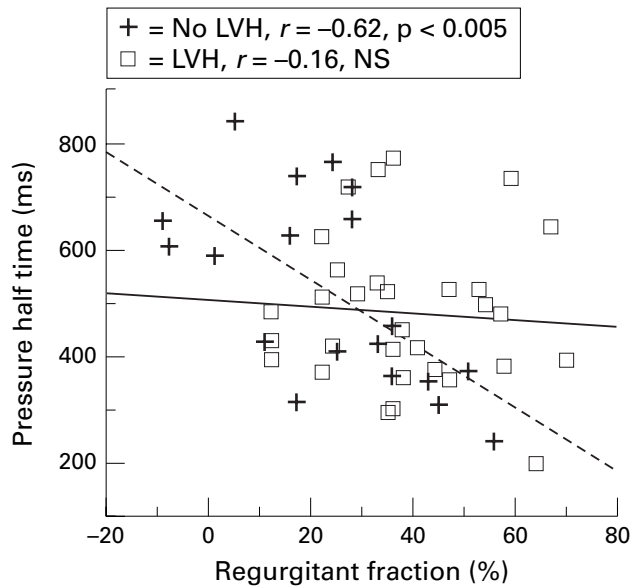

Figure 2 Correlation between the regurgitant fraction and the pressure half time in patients with normal $(+$, dashed regression line) and abnormal ( $\square$, plain regression line) left ventricular mass index. LVH, left ventricular hypertrophy.

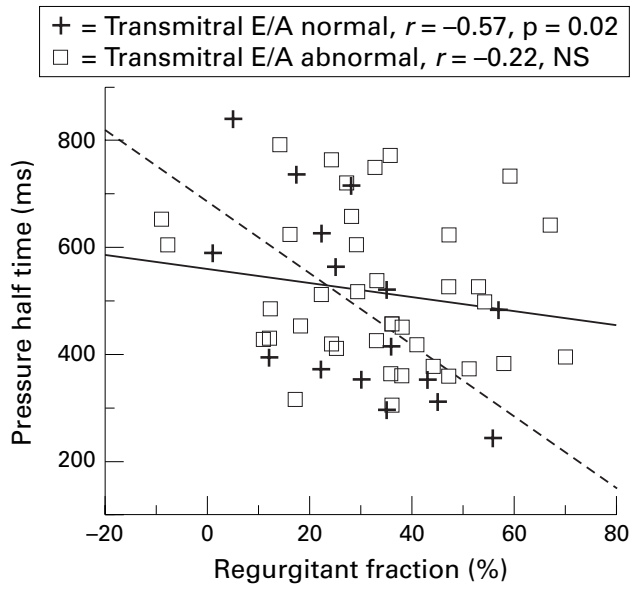

Figure 3 Correlation between the regurgitant fraction and the pressure half time in patients with normal $(+$, dashed regression line) and abnormal ( $\square$, plain regression line) transmitral E/A ratio. Transmitral E/A, early to late transmitral flow velocity ratio.

PHT $=696-6.60 \times$ regurgitant fraction; $r=0.57, \mathrm{p}=0.02$; for E-DT $<220 \mathrm{~ms}$ : $\mathrm{PHT}=611-4.28 \times$ regurgitant fraction; $r=0.50, \quad \mathrm{p}<0.001 ;$ for $\mathrm{E} / \mathrm{ADTI}<1$ : $\mathrm{PHT}=569-4.26 \times$ regurgitant fraction; $r=0.57, \mathrm{p}=0.02$, figs $3-5)$. These correlations were closer than the overall correlation between the regurgitant fraction and the PHT. The slope of the regurgitant fraction to PHT relation was similar in all normal groups (dashed lines). With increasing aortic regurgitation severity, the PHT tended to diverge between normal and abnormal groups. The PHT tended to remain increased in the groups with left ventricular hypertrophy and relaxation abnormalities, whereas it decreased significantly in the normal groups.

Using the pulmonary venous flow parameters and the left ventricular relaxation constant $\tau$, no differences were found between normal and abnormal groups in terms of the regurgitant fraction to PHT relation.

\section{Discussion}

This study in patients with aortic regurgitation confirms data from previous experimental and

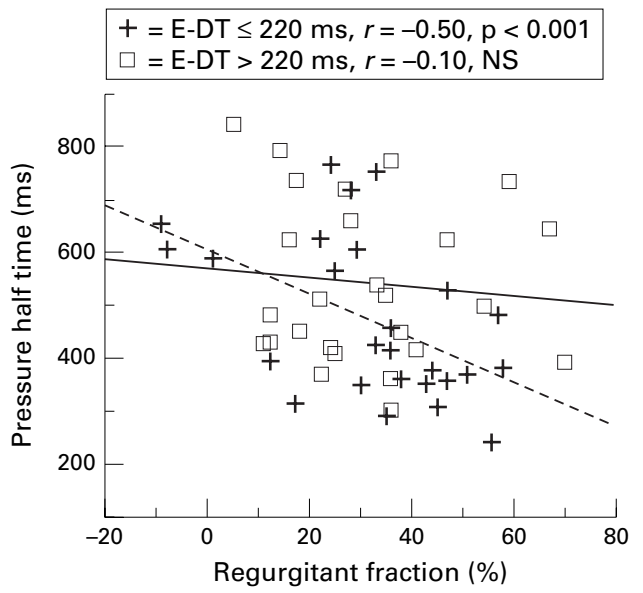

Figure 4 Correlation between the regurgitant fraction and the pressure half time in patients with a deceleration time of early transmitral filling $\leqslant 220 \mathrm{~ms}(+$, dashed regression line) and $>220 \mathrm{~ms}$ ( $\square$, plain regression line). E-DT, deceleration time of early transmitral filling.

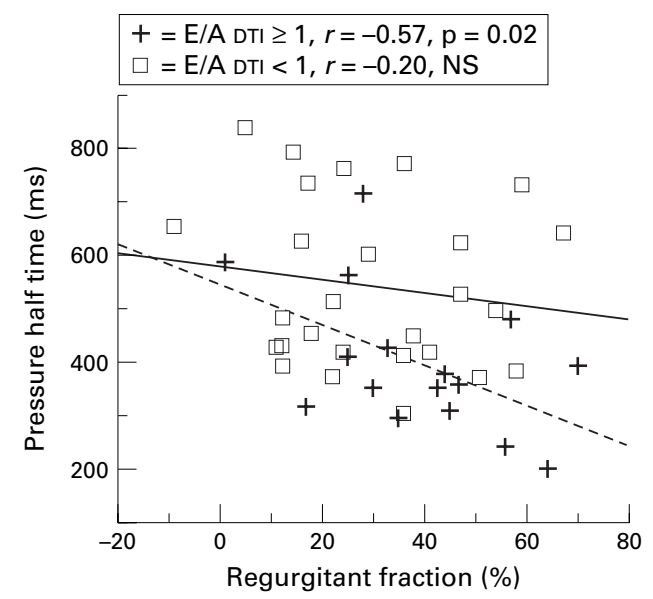

Figure 5 Correlation between the regurgitant fraction and the pressure half time in patients with an early to late diastolic mitral annulus peak motion velocity ratio of $\geqslant 1$ $(+$, dashed regression line) and $<1$ ( $\square$, plain regression line). E/A DTI, early to late diastolic mitral annulus peak motion velocity ratio assessed by Doppler tissue imaging.

mathematical models showing that the diastolic properties of the left ventricle substantially influence the PHT of the regurgitant Doppler spectrum. In the presence of impaired left ventricular relaxation, the pressure or velocity decay of aortic regurgitation is not related to its severity. This probably results from widely varying prolongation of the PHT in patients with different degrees of disturbed left ventricular relaxation for a given degree of aortic regurgitation. Conversely, in normal left ventricular relaxation, the PHT significantly decreases with increasing aortic regurgitation severity.

INFLUENCE OF VASCULAR AND VENTRICULAR

FACTORS ON THE PRESSURE HALF TIME:

PUBLISHED REPORTS

The PHT is a quantitative parameter of the pressure equilibration rate between aorta and left ventricle. This pressure equilibration is not only influenced by the regurgitant orifice area alone, but also by variables affecting the diastolic pressure decay in the aorta and the diastolic pressure rise in the left ventricle. 
When these two pressure curves rapidly converge during diastole the PHT is short, and vice versa. The influence of aortic compliance, systemic vascular resistance, and left ventricular compliance have previously been studied in mathematical and experimental models by Griffin et al and Slordahl et al. ${ }^{8911}$ A high systemic vascular resistance has been shown to increase regurgitation severity and simultaneously to prolong the PHT. ${ }^{9}$ On the other hand, increasing the aortic compliance diminishes regurgitation severity while prolonging the PHT. The present study accounted only indirectly for aortic compliance and diastolic vascular resistance by measuring the end diastolic diameter of the aorta and diastolic blood pressure. Those variables, together with cardiac output, did not differ between the study groups (tables 1 and 2), indicating that aortic compliance and systemic vascular resistance did not have much influence on the variable results found in patients with and without left ventricular hypertrophy. Decreasing the compliance of the left ventricle has been shown in vitro to shorten the PHT. ${ }^{811}$ Teague et al have shown that patients with restrictive ventricular physiology had a shortened PHT within each of four aortic regurgitation severity groups. ${ }^{7}$ However, the influence of left ventricular relaxation, another aspect of diastolic function, on the PHT has never been investigated in humans.

INFLUENCE OF LEFT VENTRICULAR RELAXATION PROPERTIES ON THE PRESSURE HALF TIME

In this study we showed that PHT tended to remain elevated despite increasing severity of aortic regurgitation in patients with left ventricular hypertrophy and left ventricular relaxation abnormalities. These consistent findings warrant interpretation as they apparently contradict in vitro investigations that showed a shortened PHT in the presence of decreased left ventricular compliance. ${ }^{89}{ }^{11}$ The principal difference between the present study and those in vitro model studies lies in the divergent focus on left ventricular relaxation abnormalities and reduced left ventricular compliance- - two different features of diastolic dysfunction. ${ }^{23}$ In analogy to early transmitral flow velocity profiles in the presence of impaired relaxation and severely diminished left ventricular compliance, with prolonged and shortened $\mathrm{E}$ wave deceleration time respectively, "aortic regurgitation deceleration" (pressure half time) tends to be extended in abnormal relaxation, whereas it is shortened in (severely) stiff ventricles. Haemodynamically, the presence of abnormal left ventricular relaxation causes the early diastolic left ventricular pressure drop to be prolonged, thus delaying the pressure rise later into diastole when relaxation is complete. In aortic regurgitation, and compared with the situation where left ventricular relaxation is normal, the diastolic pressure difference between aorta and left ventricle falls more slowly when there is abnormal left ventricular relaxation-that is, the PHT is prolonged. Because in abnormal left ventricular relaxation left ventricular compliance may also be dimin- ished, the net effect of the two counteracting forces on the PHT may vary widely, reflecting the continuum of transmitral flow velocity profiles in diastolic dysfunction that change from reduced, prolonged early filling, to "pseudonormal," to restrictive filling patterns. ${ }^{24}$ This variability in PHT is clearly reflected in the extended scatter of the variables among patients with left ventricular hypertrophy or disturbed left ventricular relaxation (figs 2-5). It is responsible for the fact that there is no association between the regurgitant fraction and PHT in this group of patients with aortic regurgitation, and thus that this method cannot be used for assessment of aortic regurgitation in patients with left ventricular hypertrophy.

The assessment of the left ventricular relaxation constant $\tau$ from the upstroke of the aortic regurgitation Doppler signal profile was performed to attempt a quantification of left ventricular relaxation and not just a qualitative assessment in analogy to invasive procedures. In contrast to other recently published studies, ${ }^{18}$ however, problems such as imperfect border delineation of the Doppler velocity upstroke in aortic regurgitation made it impossible to perform this measurement reliably.

RESTRICTIONS OF THE INFLUENCE OF LEFT VENTRICULAR RELAXATION ABNORMALITIES ON THE PRESSURE HALF TIME

A decreasing overlap of the PHT between the groups with more severe aortic regurgitation (figs 2-5) suggests that in mild aortic regurgitation, PHT is predominantly influenced by the regurgitant orifice size, whereas in severe aortic regurgitation a PHT prolonging effect of a relaxation abnormality is unmasked. This effect can be compared with the haemodynamically similar situation in mitral stenosis. In both settings, the left ventricle is (initially) passively filled through a small orifice, and the PHT of the passive filling is an index of the severity of the orifice narrowing. ${ }^{25-28}$ However, the milder the mitral stenosis-that is, the larger the orifice area-the shorter becomes the PHT of the early transmitral filling, and the greater becomes the influence of left ventricular relaxation on the PHT. If left ventricular relaxation is impaired, the PHT of the early filling remains prolonged even in very mild mitral stenosis. Similarly, our data show that the influence of the left ventricular relaxation abnormality on the PHT is unmasked in severe aortic regurgitation, that is, in large orifice areas.

CLINICAL IMPLICATIONS

The PHT method for the quantitative assessment of aortic regurgitation has important limitations that should be considered. Probably the most important restriction to its usefulness for clinical decision making is the influence of an abnormal left ventricular relaxation on the PHT. First, disturbed relaxation of the left ventricle is a situation very frequently encountered in aortic regurgitation, especially in chronic aortic regurgitation. Second, the prolonging influence of disturbed left ventricular relaxation on the PHT may lead to considerable 
underestimations of the severity of aortic regurgitation. Third, this influence is predominantly observed in moderate and severe aortic regurgitation (the clinically most relevant situation). In order to prevent underestimations, the PHT method should not be employed in the presence of left ventricular hypertrophy and aortic stenosis, as abnormal relaxation can be expected in these patients. If the echo quality is good and if cardiac shunts and other than aortic valve disease are excluded, the Qp/Qs method should be used. ${ }^{4}$ Alternatively, the vena contracta method ${ }^{29}$ or Doppler echocardiography of the descending aorta, ${ }^{30} 31$ in addition to colour Doppler echocardiography, can be used. During our study protocol, the proximal isovelocity surface area method (PISA) for the assessment of the aortic regurgitation ${ }^{32}{ }^{33}$ was not applicable in most patients because of the relatively small proximal chamber size (aortic root) and because of frequent artefacts close to valve calcifications.

\section{STUDY LIMITATIONS}

A major limitation of the study is the fact that the $\mathrm{Qp} / \mathrm{Qs}$ method used as a gold standard for the assessment of aortic regurgitation includes measurements that are prone to errors. Poor lateral resolution may affect the measurement of the pulmonary artery diameter. The interobserver variability was therefore relatively high. Negative values for regurgitant fraction are the result of this variability in patients with mild aortic regurgitation. Planimetry of the diastolic aortic valve orifice area was not performed because of frequent artefacts caused by valve calcifications. The visual linear fitting of the aortic regurgitation flow velocity curve is another (less important) source of error. The PHT measurement is most accurate in steep aortic regurgitation flow velocity curves, whereas in flat curves the PHT measurement is very sensitive to small changes in the visual fitting. This may explain the higher PHT variability with a small regurgitant fraction than with severe aortic regurgitation. Our study design did not include invasive left ventricular pressure measurements, which would have provided the gold standard for the assessment of left ventricular relaxation.

\section{CONCLUSIONS}

There is a considerable influence of left ventricular relaxation abnormalities on the pressure half time of aortic regurgitation. From echocardiographic assessment, the magnitude of this influence is unpredictable because of methods for measuring different left ventricular relaxation abnormalities are lacking. We conclude that the PHT method for the assessment of aortic regurgitation should only be used in patients with pure aortic regurgitation, normal left ventricular mass, and normal left ventricular function.

1 Henry WL, Bonow RO, Borer JS, et al. Observations on the optimum time for operative intervention for aortic regurgioptimum time for operative intervention for aortic regurgireplacement in symptomatic patients. Circulation replacement in
1980;61:471-83

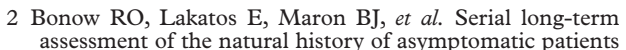
assessment of the natural history of asymptomatic patients with chronic aortic regurgitation and normal left vent
lar systolic function. Circulation 1991;84:1625-35.

3 Carabello BA, Usher BW, Hendrix GH, et al. Predictors of outcome for aortic valve replacement in patients with aortic regurgitation and left ventricular dysfunction: a change in the measuring stick. F Am Coll Cardiol 1987;10:991-7.

4 Kitabatake $\mathrm{A}$, Ito $\mathrm{H}$, Inoue $\mathrm{M}$, et al. A new approach to noninvasive evaluation of aortic regurgitant fraction by two-dimensional Doppler echocardiography. Circulation 1985;72:523-9.

5 Enriquez-Sarano M, Bailey KR, Seward JB, et al. Quantitative Doppler assessment of valvular regurgitation. Circulation 1993;87:841-8.

6 Labovitz AJ, Ferrara RP, Kern MJ, et al. Quantitative evaluation of aortic insufficiency by continuous wave Doppler ation of aortic insufficiency by continuous wave Dopp

7 Teague SM, Heinsimer JA, Anderson JL, et al. Quantification of aortic regurgitation utilizing continuous wave Doption of aortic regurgitation utilizing continuous wave
pler ultrasound. $\mathcal{F}$ Am Coll Cardiol 1986;8:592-9.

8 Griffin BP, Flachskampf FA, Siu S, et al. The effects of regurgitant orifice size, chamber compliance, and systemic vascular resistance on aortic regurgitant velocity slope and pressure half-time. Am Heart f 1991;122:1049-56

9 Griffin BP, Flachskampf FA, Reimold SC, et al. Relationship of aortic regurgitant velocity slope and pressure half-time to severity of aortic regurgitation under changing haemodynamic conditions. Eur Heart f 1994;15:681-5.

10 Samstad SO, Hegrenaes L, Skjaerpe T, et al. Half time of the diastolic aortoventricular pressure difference by continuous wave Doppler ultrasound: a measure of the severity of aortic regurgitation? Br Heart $\mathcal{f}$ 1989;61:336-43.

11 Slordahl SA, Piene H, Skjaerpe T. Pressure half-time in aortic regurgitation: evaluation with Doppler in a cardiovascular hydromechanical simulator and in a computer model. f Am Soc Echocardiogr 1990;3:46-53.

12 Sohn DW, Chai IH, Lee DJ, et al. Assessment of mitral annulus velocity by Doppler tissue imaging in the evaluation of left ventricular diastolic function. $\mathcal{F} \mathrm{Am}$ Coll Cardiol 1997;30:474-80

13 Sutherland GR, Stewart MJ, Groundstroem KW, et al. Color Doppler myocardial imaging: a new technique for the assessment of myocardial function. F Am Soc Echocardiogr 1994;7:441-58.

14 McDicken WN, Sutherland GR, Moran CM, et al. Colour Doppler velocity imaging of the myocardium. Ultrasound Med Biol 1992;18:651-4.

15 Miyatake K, Yamagishi M, Tanaka N, et al. New method for evaluating left ventricular wall motion by color-coded tissue Doppler imaging: in vitro and in vivo studies. $\mathcal{f} \mathrm{Am}$ Coll Cardiol 1995;25:717-24.

16 Mantero A, Gentile F, Gualtierotti C, et al. Left ventricular diastolic parameters in 288 normal subjects from 20 to 80 years old. Eur Heart f 1995;16:94-105.

17 Nagueh SF, Middleton KJ, Kopelen HA, et al. Doppler tissue imaging: a noninvasive technique for evaluation of left ventricular relaxation and estimation of filling pressures. $\mathcal{F}$ Am Coll Cardiol 1997;30:1527-33.

18 Yamamoto K, Masuyama T, Doi Y, et al. Noninvasive assessment of left ventricular relaxation using continuouswave Doppler aortic regurgitant velocity curve: its comparative value to the mitral regurgitation method. Circulation 1995;91:192-200.

19 Sahn DJ, De Maria A, Kisslo J, et al. Recommendations regarding quantitation in M-mode echocardiography: results of a survey of echocardiographic measurements. Circulation 1978;58:1072-83.

20 Troy BL, Pombo J, Rackley CE. Measurement of left ventricular wall thickness and mass by echocardiography. Circulation 1972;45:602-11.

21 Devereux RB, Lutas EM, Casale PN, et al. Standardization of $\mathrm{M}$-mode echocardiographic left ventricular anatomic measurements. F Am Coll Cardiol 1984;4:1222-30.

22 Gentile F, Mantero A, Lippolis A, et al. Pulmonary venous flow velocity patterns in 143 normal subjects aged 20 to 80 years old. An echo 2D colour Doppler cooperative study. Eur Heart f 1997;18:148-64.

23 Noble MI, Milne EN, Goerke RJ, et al. Left ventricular filling and diastolic pressure-volume relations in the conscious dog. Circ Res 1969;24:269-83.

24 Nishimura RA, Tajik AJ. Evaluation of diastolic filling of left ventricle in health and disease: Doppler echocardiography is the clinician's Rosetta Stone. F Am Coll Cardiol 1997;30: $8-18$.

25 Wranne B, Ask P, Loyd D. Analysis of different methods of assessing the stenotic mitral valve area with emphasis on the pressure gradient half-time concept. Am f Cardiol 1990;66:614-20.

26 Hatle L, Angelsen B, Tromsdal A. Noninvasive assessment of atrioventricular pressure half-time by Doppler ultrasound. Circulation 1979;60:1096-104

27 Smith MD, Wisenbaugh T, Grayburn PA, et al. Value and limitations of Doppler pressure half-time in quantifying mitral stenosis: a comparison with micromanometer catheter recordings. Am Heart f 1991;121:480-8.

28 Thomas JD, Weyman AE. Doppler mitral pressure halftime: a clinical tool in search of theoretical justification. $\mathcal{f}$ Am Coll Cardiol 1987;10:923-9.

29 Ishii M, Jones M, Shiota T, et al. Evaluation of eccentric aortic regurgitation by color Doppler jet and color aortic regurgitation by color Doppler jet and color study of quantified aortic regurgitation. Am Heart $\mathcal{f} 1996$; 132:796-804. 
30 Touche T, Prasquier R, Nitenberg A, et al. Assessment and follow-up of patients with aortic regurgitation by an updated Doppler echocardiographic measurement of the regurgitant fraction in the aortic arch. Circulation 1985;72: 819-24.

31 Boughner DR. Assessment of aortic insufficiency by transcutaneous Doppler ultrasound. Circulation 1975;52:874-9.
32 Utsunomiya T, Ogawa T, Tang HA, et al. Doppler color flow mapping of the proximal isovelocity surface area: a new method for measuring volume flow rate across orifice. F Am Soc Echocardiogr 1991;4:338-48.

33 Giesler M, Grossmann G, Schmidt A, et al. Color Doppler echocardiographic determination of mitral regurgitant flow from the proximal velocity profile of the flow convergence region. Am f Cardiol 1993;71:217-24.

\section{IMAGES IN CARDIOLOGY}

\section{Pitfall in the diagnosis of pericardial effusion by echocardiography}

A 65 year old man with an 18 year history of ischaemic heart disease was admitted with a six week history of severe weakness and orthostatic hypotension. Eight weeks before admission he underwent echocardiography at his local hospital to evaluate exertional dyspnoea; pericardial effusion was suspected. Because of poor ultrasound conduction, ventricular or valvar function could not be evaluated. The patient was treated with diuretics; however, symptoms did not improve and the diuretics were increased to a maximum of $750 \mathrm{mg}$ frusemide daily.

On admission there were no clinical findings of heart failure. The patient had severe orthostatic hypotension and felt vertiginous. Transthoracic echocardiography (A) revealed nor mal systolic left ventricular function and thickened left ventricular wall. The subepicar-dial space was enlarged to about $2 \mathrm{~cm}$, which appeared not to be completely free from echos suggesting chronic pericardial effusion. Magnetic resonance imaging (B) and computed tomography revealed density of the subepicardial space from fat tissue.

Massive subepicardial lipomatosis should be considered in the differential diagnosis of pericardial effusion suspected by echocardiography, especially in case of poor ultrasound conduction. If there is still doubt, further examination with computed tomography or magnetic resonance imaging should be considered. (ES, epicardial space; LV, left ventricle; LA, left atrium; $\mathrm{AO}$, aortic root; $\mathrm{RV}$, right ventricle.)
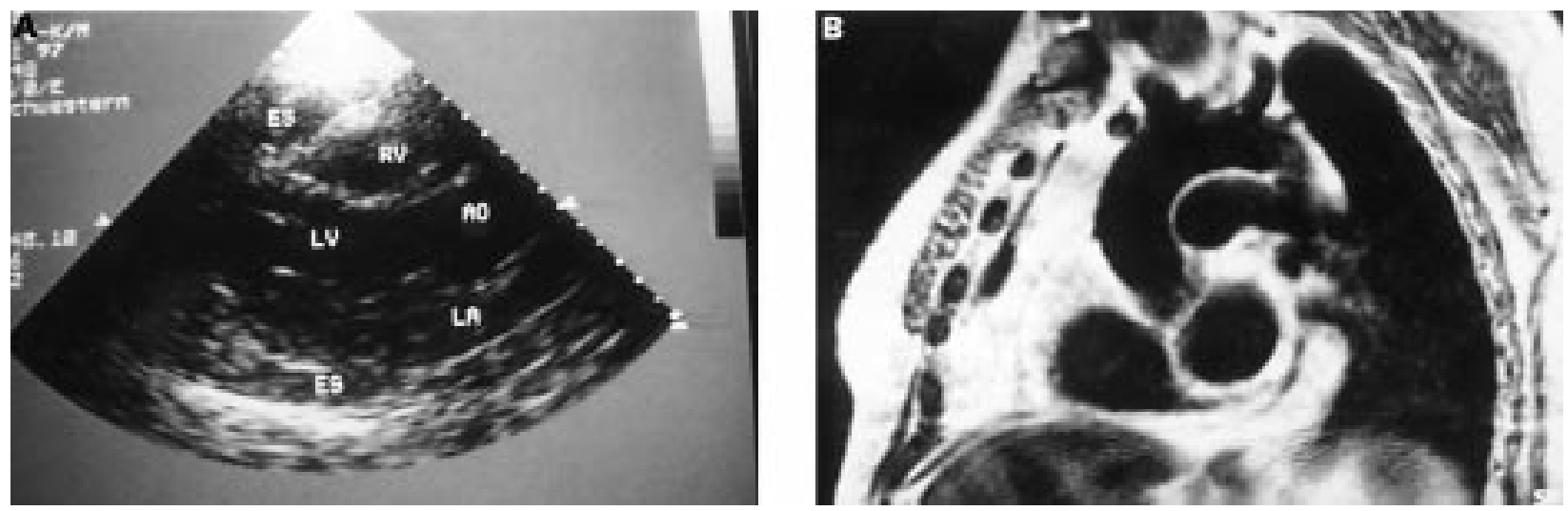

J AUER

R BERENT

B EBER 